umol/L (reference range $<0.32 \mathrm{umol} / \mathrm{L}$ ). Intrinsic factor and gastric parietal cell antibodies were detected.

Conclusion Our patient had a clinical presentation consistent with B12 deficiency with an erroneously high active B12 level. Functional assays confirmed B12 deficiency, and a serological diagnosis of pernicious anaemia was made. This case illustrates the importance of not relying on any single test to exclude B12 deficiency.

\section{PARANEOPLASTIC PROGRESSIVE-SUPRANUCLEAR PALSY LIKE BRAINSTEM SYNDROME ASSOCIATED WITH LUNG ADENOCARCINOMA}

${ }^{1}$ Natasha Gerbis, ${ }^{2}$ Ariadna Fontes-Villalba, ${ }^{3,4}$ Patrick Aouad, ${ }^{2,3}$ Suran Fernando, ${ }^{2,3}$ John DE Parratt. 'Northern Beaches Hospital, Frenchs Forest, NSW, Australia; ${ }^{2}$ Royal North Shore Hospital, St Leonards, NSW, Australia; ${ }^{3}$ University of Sydney, Sydney, NSW, Australia; ${ }^{4}$ Liverpool Hospital, Sydney, NSW, Australia

\subsection{6/bmino-2021-ANZAN.116}

Objectives Progressive supranuclear palsy (PSP) is a neurodegenerative condition characterised by Parkinsonian features, cervical dystonia and ophthalmoparesis. Paraneoplastic PSP has previously been reported in the literature in association with several different cancer types but is very rare. ${ }^{1-3}$

Methods Case review.

Results A 74 year old Chinese man was diagnosed with Stage 1b EGFR positive lung adenocarcinoma and underwent a left upper lobectomy. Twelve months later he presented with rapidly progressive neck stiffness, reduction in motor function and gait (over 8 weeks) and recalcitrant disequilibrium.

$\mathrm{He}$ had hypomimia, frontalis over-activation, blepharospasm, blepharotremor and a supranuclear gaze palsy. There was marked axial rigidity, bradykinesia and cervical dystonia. An MRI brain and spine were unremarkable. Vestibular function tests were normal. Serum antineuronal antibodies were negative. The CSF analysis was unremarkable.

The patient responded to plasma exchange on a two to three-weekly basis with significant improvement in saccadic eye movements and Parkinsonism. However, disequilibrium remained a persistent problem despite the discovery and excision of a second EGFR wild type non-small cell lung cancer, and Rituximab was recently started. Cervical dystonia was treated partially with Botulinum toxin injections, but the patient responded poorly to L-dopa.

Conclusions This suspected paraneoplastic disease exhibits several features of PSP. In particular, the supranuclear palsy, Parkinsonism and dystonia are similar to the typical syndrome. However, the rapidly progressive presentation and disequilibrium are unusual and a response to plasma exchange, suggests humorally mediated neuronal pathology. In rapidly evolving PSP-like cases with cancer, investigation for immunopathology is warranted.

\section{REFERENCES}

1. Dash D, Choudhary R, Ramanujam B, Vasantha PM, Tripathi M. Paraneoplastic syndrome mimicking progressive supranuclear palsy. I Clin Neurosci 2016
Oct;32:162-3. doi:10.1016/j.jocn.2016.02.032. Epub 2016 Jun 16. PMID: 27318371.

2. Takkar A, Mehta S, Gupta N, Bansal S, Lal V. Anti- RI antibody associated progressive supranuclear palsy like presentation in a patient with breast carcinoma. $J$ Neuroimmunol 2020 Oct 15;347:577345. doi:10.1016/j.jneuroim.2020.577345. Epub 2020 Jul 26. PMID: 32763584.

3. Tan JH, Goh BC, Tambyah PA, Wilder-Smith E. Paraneoplastic progressive supranuclear palsy syndrome in a patient with B-cell lymphoma. Parkinsonism Relat Disord 2005 May;11(3):187-91. doi:10.1016/j.parkreldis.2004.09.003. PMID: 15823484.

\section{7 MULTIPLE SCLEROSIS PRESENTING AS A RAPIDLY PROGRESSIVE DEMENTIA}

${ }^{1}$ Natasha Gerbis, ${ }^{2,3}$ John DE Parratt. 'Northern Beaches Hospital, Frenchs Forest, NSW, Australia; ${ }^{2}$ Royal North Shore Hospital, St Leonards, NSW, Australia; ${ }^{3}$ University of Sydney, Sydney, NSW, Australia

\subsection{6/bmjno-2021-ANZAN.117}

Objectives Rapidly progressive dementias manifest with cognitive decline that progresses over weeks to months. It is rare for dementia to present early in the course of multiple sclerosis (MS). However, several case series on rapidly progressive dementia include a small number of patients with MS, and one small case series specifically examined dementia presenting within five years of MS diagnosis.

Methods Case study.

Results A 66 year old woman presented to hospital with progressive left upper limb weakness and subacute cognitive decline over six to nine months. MRI brain revealed a right frontoparietal tumefactive lesion. Oligoclonal bands were detected in the CSF and a brain biopsy confirmed demyelination. The patient's serum was tested in our research lab and revealed a novel antibody.

The patient experienced a fluctuating clinical course but responded well to treatment with intravenous (IV) methylprednisolone, IV immunoglobulin (IVIG) and Cyclophosphamide. Unfortunately, further deterioration occurred five months after treatment with Cyclophosphamide and Rituximab was initiated. Clinical improvement in cognition was again observed, albeit briefly.

Six months later, the patient died due to bronchopneumonia. The period from initial presentation to death spanned 30 months. A non-coronial autopsy was performed and revealed widespread incomplete cortical myelin loss and atrophy.

Conclusions Although a few cases have previously been reported in the published medical literature, dementia in the early stages of MS is extremely rare and may in fact be due to a distinct immunopathologic process.

\section{REFERENCE}

1. Zhang $W$, et al, Analysis of clinical features for 8 patients with autoimmune dementia, Zhonghua Yi Xue Za Zhi. 2014 Feb 11;94(5):359-63.

2. Mendes MF, et al. Early and severe cognitive impairment in multiple sclerosis. Dement Neuropsychol 2012 Jan-Mar;6(1);48-52. 